\title{
EXTENSIVE BACK ULCERATION DUE TO PAINTBALL PELLET INJURY - A CASE REPORT OF 24-YEAR-OLD MAN
}

\author{
Paweł Brzewski ${ }^{1(\mathrm{~A}, \mathrm{~B}, \mathrm{D}, \mathrm{E})}$, Katarzyna Malec $^{2(\mathrm{~A}, \mathrm{~B}, \mathrm{E}, \mathrm{F})}$, Joanna Sułowicz $^{1(\mathrm{~A}, \mathrm{D})}$, Anna Wojas-Pelc ${ }^{1(\mathrm{~A}, \mathrm{~B}, \mathrm{D})}$ \\ ${ }^{1}$ Department of Dermatology Collegium Medicum UJ, Krakow, Poland \\ ${ }^{2}$ Joseph Dietl Hospital, Krakow, Poland
}

\begin{abstract}
Paintball is a kind of a team game, depending on simulated fight with the use of the devices similar in shape and principle to the pneumatic weapon, called markers. Balls filled with paint, made on base of food gelatin, are thrown out with the help of the compressed air, propane or carbon dioxide. A direct hit on an enemy, during which the ball crashes and leaves a colorful trace (called by players splat), means elimination from the game.

We would like to present a case report of a 24-year-old man, who was shot by two pellets in the area between scapular bones from a close distance (about 4 meters $\approx 13,12$ feet). The victim described a strong burning sensation, pain and skin lesion. The laboratory tests performed during the same visit were within reference range.

During the follow-up examination after one month the wound evolved into a marginal epithelialization with a superficial necrolysis in the center. The one year follow-up visit showed marginal cicatrisation and central crust indicating final stages of re-epithelium fibrosis and healing in the place of the injury. During the follow-up examination two years after the event, patient's main complaints were itching and hyperesthesia in the surrounding of the lesion. An extensive scar between scapulas has been formed in the place of initial injury.

The potentially safe team game with typical, expected type of injury caused at the young healthy man the significant deformation of the skin.
\end{abstract}

Key words: paintball injury, pellet injury, skin ulceration

\section{Introduction}

Paintball guns and pellets were originally invented and patented in 1970 by James Hale and used for marking trees for excavation and cuttles. The first use of this non-powder gun for recreational purpose took place eleven years later-twelve players competed against each other with Nel-spot 007 pistols. The first outdoor paintball field was opened in Rochester, N.Y. in 1982. At the beginning of ' 90 this type of survival game became popular in Europe [1]. In this combat simulating sport players compete, in teams or individually, to eliminate opponents by hitting them with special bullets. When pellets strike participants, they are marked with paint. The standard bullet is composed of a thin gelatin capsule filled with non-toxic and water-soluble substances, has a diameter of approximately $17 \mathrm{~mm}$ ( 0.68 caliber), an average weight of 3.39 , with a very thin shell to guarantee breaking upon impact. Balls are fired by compressed gas-driven guns at up to 85 $\mathrm{m} / \mathrm{s}$ (what is approximately $300 \mathrm{~km} / \mathrm{h}$ ).

When paintball became more popular, especially among young people, the number of injuries related to this game increased. A primary health concern regarding paintball game related sports has been the risk of injury to the eye [2]. Numerous case studies have reported on ocular injuries resulting from a paintball [3]. When projectile hit the eye, can cause severe damage leading to reduced visual acuity or even blindness [4].
Another common paintball game related injuries are the effects of overexertion or fall and the cutaneous lesions produced by pellets $[2,5]$. Travelling at such high speeds, the paintball can easily damage the skin.

The most frequent character of the cutaneous lesion is purpura. Purpura caused by paint pellets was first reported by Siegel et al. [6] and named „paint pellet purpura". Subsequently, Rahbari and Nabai [7] described similar case and named it „paint pellet erythema”. Also Levsky and Crow [8] reported case of annular erythematous patches produced by projectile.

\section{Case report}

We describe a case of 24-year-old male who presented in the Department of Dermatology with the lesion on his back. Upon questioning, he reported participating in paintball game on previous day. The lesion was the result of the skin being struck by a rapidly moving paint-ball containing a green liquid. The pellet was fired from the distance of approximately $4 \mathrm{~m}$ and hit the skin through a thick military jacket and a cotton T-shirt. The lesion consisted of butterflyshaped superficial ulceration (approximately $10 \mathrm{~cm} \mathrm{x}$ $5 \mathrm{~cm}$ ) with erythematous well-defined borders. It was formed on skin of the back, alongside the vertebra, between scapulas (Fig. 1 presents butterfly-shaped skin ulceration covered with crust. In the left lower back typical paintball purpura can be seen.). There was no 


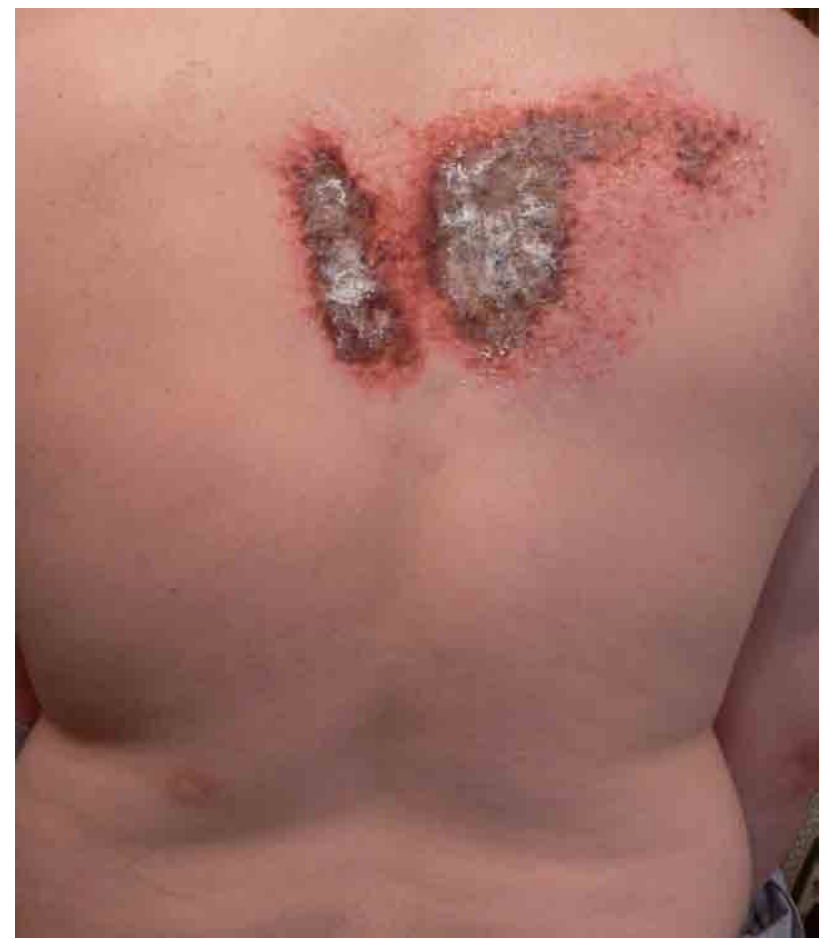

Fig 1. Butterfly-shaped skin ulceration covered with crust. In the left lower back typical paintball purpura.

sign of the green pigment on the skin surface. The patient had non-significant medical history. Laboratory evaluation was within normal limits. The injured area was treated with the silver sulfadiazine ointment and rivanol-salicylates ointment.

During follow-up examination (approximately one month after the day of the injury) slight epithelialization from the borders of ulceration has been noticed. In the center of the injury superficial tissue loss still remained. After one year, in the area of initial lesion marginal cicatrisation and central crust (indicating final stages of re-epithelium fibrosis and healing) have been formed. During the follow-up examination two years after the event, patient's main complaints were itching and hyperesthesia in the surrounding of the initial injury. Fig. 2 illustrates extensive scar between scapulas remaining after two years. The patient still remains in dermatological care.

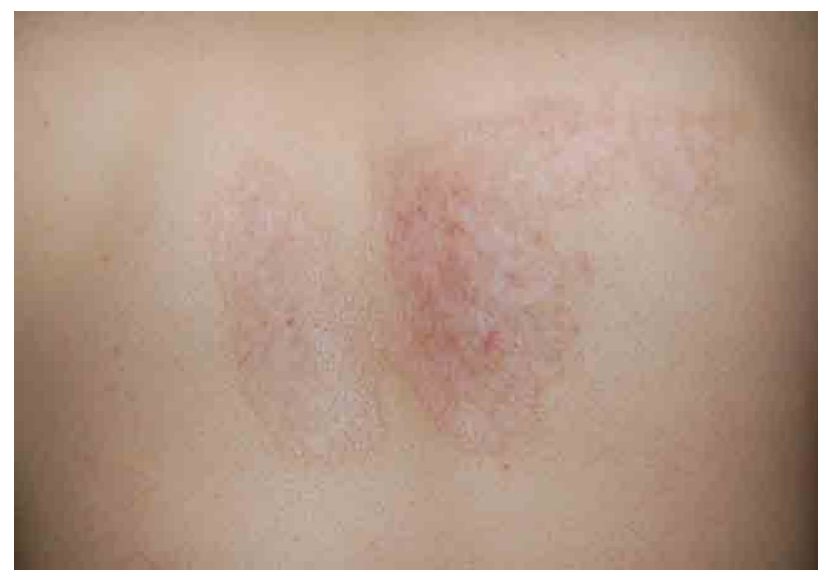

Fig 2. Two-year follow-up after paintball lesion-extensive scar between scapulas

\section{Discussion}

We believe that this is the first case of such severe skin injury due to paint pallet. There are published a few cases of paintball purpura and ocular injuries but none about skin ulceration after being struck by a paint projectile. The pathophysiology of this severe and rapidly shaping ulceration remains unclear. We believe that the histopathological examination of tissues sample from the injured area would be very valuable and desirable source of information but patient did not consent.

The contact allergic reaction has been considered. However, taking into consideration that the green paint in the ball was food pigment and patient was not allergic to this type of dye, it is unlikely.

Also mechanical injury was considered, but as in other areas of the body typical injuries (bruises, erythema) has been found, it seems to be of a little probability.

Patient's past medical history was non-significant and laboratory evaluation was within normal limits therefore we do not suspect that this skin ulceration is due to some different systemic disease. Also pyoderma gangrenosum need to be considered as a cause of this cutaneous ulceration. This lesion could be a manifestation of the phenomenon called pathergy which can be observed in this disease [9]. Against this diagnosis stand: non-typical age for pyoderma gangrenosum (predominantly occurs in the fourth and fifth decades of life), absence of extracutaneous changes or systemic illness (it is associated with systemic diseases in at least $50 \%$ of patients who are affected) and the fact that skin ulceration in this case healed without typical treatment $[10,11]$. However in that case pyoderma gangrenosum cannot be completely rejected because this is a diagnosis of exclusion and no specific criteria have been determined to confirm the diagnosis [12].

\section{Limitations of the article}

The most important limitation of this paper is lack of histopathological examination. The reason why this examination was not proceed is the fact that the patient did not consent. We strongly believe that the microscopic examination of biopsy would be very valuable and desirable source of information.

\section{References}

1. Amodea J. History of paintball. Available at: http://www.pcri. net/history.htm . Accessed July 29, 2002.

2. Conn JM, Annest JL, Gilchrist J et al. Injuries from paintball game related activities in the United States, 1997-2001. Injury Prevention 2004; 10: 139-43.

3. Taban M, Taban M, Sears JE. Ocular findings following trauma from paintball sports. Eye (Lond) 2008; 22 (7): 930-4.

4. Greven CM, Bashinsky AL. Circumstance and Outcome of Ocular Paintball Injuries. Am J Ophthalmol 2006; 141 (2): 393.

5. Ambay AR, Stratman EJ. Paintball: dermatologic injuries. Cutis 2007; 80 (1): 49-50. 
6. Siegel DM, Goldberg LH, Altman AR et al. Paint Pellet Purpura: A Peril for Pistol-Packing Paramilitary Personnel. JAMA 1986; 255: 3367.

7. Rahbari H, Nabai H. Paint pellet erythema. Pediatr Dermatol 1996; 13: 174-5.

8. Levsky ME, Crowe M. What is your diagnosis? Paintball purpura. Cutis 2005; 75:148, 157-8.

9. Lindberg-Larsen R, Fogh K. Traumatic pyoderma gangrenosum of the face: pathergy development after bike accident. Dermatology 2009; 218(3): 272-4.

10. Saigal R, Singh Y, Mittal $M$ et al. Pyoderma gangrenosum. J Assoc Physicians India 2010; 58: 378-83.

11. Wollina U, Haroske G. Pyoderma gangrenosum. Curr Opin Rheumatol 2011;23(1): 50-6.

12. Callen JP, Jackson JM. Pyoderma gangrenosum: an update. Rheum Dis Clin North Am 2007; 33(4): 787-802.
Received: January 01, 2011

Accepted: June 01, 2011

Published: June 13, 2011

Address for correspondence:

Paweł Brzewski

Department of Dermatology Collegium Medicum UJ

31-066 Kraków

Skawińska str.8

Poland

Paweł Brzewski: brzewski@gmail.com

Katarzyna Malec: malec.katarzyna@gmail.com

Joanna Sułowicz: sulowicz@interia.pl

Anna Wojas-Pelc: wojaspelca@su.krakow.pl 